\title{
SÍNTESE DE BIODIESEL A PARTIR DE ÓLEO RESIDUAL DE FRITURA EM BANHO ULTRASSÔNICO
}

\section{Fausto de Souza Pagan ${ }^{1}$,Letícia Zanella Silva², Marcelo Bacci da Silva ${ }^{3}$, Deusmaque Carneiro Ferreira ${ }^{4}$ \\ 'Mestrando em Química do Programa de Pós-Graduação Multicêntrico em Química de Minas Gerais junto à Universidade Federal do Triângulo Mineiro. \\ 2Discente do curso de Engenharia Ambiental da Universidade Federal do Triângulo Mineiro. \\ ${ }^{3}$ Professor Doutor do Programa de Mestrado Profissional em Inovação Tecnológica, PMPIT. \\ ${ }^{4}$ Professor Doutor do Programa de Pós-Graduação em Ciência e Tecnologia Ambiental da Universidade Federal do Triângulo Mineiro, Uberaba, Minas Gerais, Brasil (Email:deusmaque.ferreira@uftm.edu.br).}

\section{Recebido em: 02/10/2017 - Aprovado em: 21/11/2017 - Publicado em: 05/12/2017 DOI: 10.18677/EnciBio_2017B122}

\begin{abstract}
RESUMO
O biodiesel representa uma alternativa essencial para atender à crescente demanda energética da sociedade moderna de forma sustentável. Os objetivos do presente estudo foram otimizar a síntese do biodiesel a partir do óleo residual de fritura em banho ultrassônico, determinar o custo de produção do biodiesel e caracterizar o produto obtido a partir dos parâmetros estabelecidos pela Agência Nacional do Petróleo, Gás Natural e Biocombustíveis (ANP). A matéria-prima utilizada foi o óleo residual de fritura, coletado no campus II da Universidade Federal do Triângulo Mineiro. As rotas foram definidas pelo Planejamento Composto Central (PCC), que otimizou as variáveis; massa de hidróxido de sódio, tempo de reação e as massas de etanol e metanol. Posteriormente, as amostras foram caracterizadas, analisando os parâmetros; massa específica, ponto de congelamento, índice de peróxido e índice de insaturação. As análises do biodiesel otimizado (rendimento superior a $90 \%$ ) ficaram dentro dos limites estabelecidos pela ANP. Os custos de produção do litro de biodiesel nas rotas metílica e etílica foram $R \$ 2,124$ e $R \$ 2,453$, respectivamente. O maior tempo de processo para a rota etílica (35\%) que elevou 0 custo de produção $(13,41 \%)$ do biodiesel nessa rota. A síntese de biodiesel otimizada a partir de óleo residual de fritura, em banho ultrassônico, constitui-se como um processo sustentável e economicamente viável para a produção em larga escala. Essa metodologia favorece a ampliação do uso de biodiesel (energia verde com menor custo de produção) como uma alternativa aos combustíveis à base de petróleo (óleo diesel).
\end{abstract}

PALAVRAS-CHAVE: biocombustível,otimização, ultrassom. 


\title{
SYNTHESIS OF BIODIESEL FROM WASTE COOKING OIL IN ULTRASSONIC BATH
}

\begin{abstract}
Biodiesel represents an essential alternative to meet the growing energy demand of modern society in a sustainable way. The objectives of the present study were to optimize the synthesis of biodiesel from the waste cooking oil in, ultrasonic bath, determine the biodiesel production cost and to characterize the product obtained from the parameters established by the National Agency of Petroleum, Natural Gas and Biofuels. The raw material used was the waste cooking oil, collected at Campus II of Federal University of Triangulo Mineiro. The routes were defined by the Central Compound Planning, which optimized the variables; mass of sodium hydroxide, reaction time and the masses of ethanol and methanol. Subsequently, the samples were characterized, analyzing the parameters; specific mass, freezing point, peroxide index and unsaturation index. The analysis of optimized biodiesel (yield over 90\%) was within the limits established by the National Agency of Petroleum, Natural Gas and Biofuels. The production costs of the liter of biodiesel in the methyl and ethyl routes were $R \$ 2.124$ and $R \$ 2.453$, respectively. The longer process time $(35 \%)$ for the ethyl route has raised the biodiesel production cost $(13.41 \%)$ on this route. The synthesis of biodiesel optimized from waste cooking oil in ultrasonic bath constitutes as an economically viable and sustainable process for large-scale production. This methodology favors the expansion of use of biodiesel (green energy with lower cost of production) as an alternative to petroleum based fuels (diesel oil)

KEYWORDS:biofuel, ultrassound, optimization.
\end{abstract}

\section{INTRODUÇÃO}

As pesquisas relacionadas ao uso de fontes renováveis de energia têm aumentando substancialmente devido às vantagens ambientais, como a redução de gases poluentes, biodegradabilidade e menor toxicidade em relação aos derivados fósseis (LOPEZ et al., 2007).

O biodiesel é um dos biocombustíveis de maior destaque no cenário mundial. Comumente produzido pela reação de transesterificação, óleos ou gorduras reagem com um álcool (metanol ou etanol) na presença de um catalisador básico $(\mathrm{KOH}$ ou $\mathrm{NaOH})$, ácido $\left(\mathrm{H}_{2} \mathrm{SO}_{4}\right.$ ou $\left.\mathrm{HCl}\right)$ ou enzimático, gerando ésteres de ácido graxo e glicerol (BRUNSCHWIG et al., 2012; CREMONEZ et al., 2014).

O ciclo do biodiesel corrobora com a redução das emissões de gases de efeito estufa na atmosfera, uma vez que a quantidade liberada de $\mathrm{CO}_{2}$ na produção e queima do biocombustível é inferior àquela absorvida no processo fotossintético de crescimento das oleaginosas (MOTA et al., 2009; KIAKALAIEH et al., 2013).

Em comparação com o diesel de petróleo, o biodiesel apresenta as mesmas capacidades térmicas e caloríficas, tendo como vantagens a emissão reduzida de gases estufa e particulados, renovabilidade da matéria-prima utilizada e viabilidades ambientais no processamento de destinação de resíduos (AARTHY et al., 2014). Nos processos de síntese do biodiesel, ao final da reação, duas fases são formadas, uma rica em ésteres de ácido graxo (biodiesel) e outra contendo gliceróis e excesso de catalisador (SNEHA et al., 2015).

Um possível processo para a síntese de biodiesel a partir de óleos residuais de fritura é a transesterificação alcalina em banho ultrassônico. O ultrassom apresenta onda mecânica contida no intervalo de frequência de $20 \mathrm{kHz}$ a um limite 
superior de $500 \mathrm{MHz}$ para líquidos e sólidos (MASON, 2007). As ondas de mais alta energia, $20-100 \mathrm{kHz}$, recentemente têm sido utilizadas em várias reações de sínteses em meio líquido denominado sonoquimíca, com equipamentos capazes de promover a cavitação e processar as reações químicas (KELKAR et al., 2008; MADDIKERI et al., 2014).

O efeito do ultrassom é na agitação da mistura dada por transmissão acústica, em que uma corrente macroscópica é induzida em fluxo no líquido pela absorção da onda mecânica (PAULA et al., 2017). Como qualquer onda sonora, o ultrassom comprime e alonga os espaços entre as moléculas do meio, causando uma série de perturbações que promovem a interação molecular específica, de modo que as reações químicas se processam mais rapidamente (JIANBING et al., 2006; GOLE et al., 2013).

Estima-se que $70-95 \%$ dos custos do processo de produção do biodiesel resultam da matéria-prima utilizada. Dessa forma, o óleo residual de fritura é uma alternativa relevante, pois, reduz os custos da matéria-prima entre $50-60 \%$ (SADEGHINEZHAD et al., 2014).

Discussões econômicas, técnicas e políticas em relação ao uso de oleaginosas na produção do biodiesel são frequentes em estudos de biocombustíveis em razão da competição com o cultivo de alimentos e o valor do produto final (COSTA et al., 2013). O uso de matérias-primas de baixo custo ou mesmo resíduos tem se mostrado uma boa alternativa para obtenção do biodiesel, diminuindo os custos de produção e aumentando a vantagem econômica quando comparado ao combustível oriundo dos óleos refinados (REIS et al., 2015).

Os objetivos do presente estudo foram otimizar a síntese do biodiesel a partir do óleo residual de fritura em banho ultrassônico, determinar o custo de produção do biodiesel e caracterizar o produto obtido a partir dos parâmetros estabelecidos pela ANP.

\section{MATERIAL E MÉTODOS}

O óleo residual de fritura foi coletado semanalmente, entre março e julho de 2017, no restaurante universitário do Instituto de Ciências Tecnológicas e Exatas da Universidade Federal do Triângulo Mineiro. O óleo residual coletado foi armazenado em galões plásticos de cinco litros. No total, foram coletados 18 litros de óleo. A produção do biodiesel por transesterificação em rotas etílica e metílica, utilizando hidróxido de sódio $(\mathrm{NaOH})$ como catalisador alcalino, se deu com a mistura dos reagentes em quantidades determinadas pelo planejamento experimental em um béquer de $250 \mathrm{~mL}$, submerso em banho ultrassônico.

A otimização estatística da reação de transesterificação para produção de biodiesel foi feita a partir do Delineamento Composto Central Rotacionado (DCCR). As variáveis foram estudadas com três diferentes níveis de codificação $(-1,0,1)$ e dois pontos axiais $(-\alpha,+\alpha)$. $O$ alfa de rotabilidade utilizado neste planejamento experimental foi de 1,68. As variáveis selecionadas foram; massa em gramas de hidróxido de sódio, massa em gramas metanol (PA) ou etanol $98^{\circ} \mathrm{GL}$ e tempo reacional em minutos. Com três variáveis $(n=3)$ selecionadas, o número de ensaios $\left(2^{n}\right)$ será igual a oito pontos fatoriais, mais seis pontos axiais e três pontos centrais, totalizando 17 ensaios. Os dados estatísticos coletados foram tratados no software Statistica®, versão 7.0.

Após a definição das variáveis, foram delimitados os valores máximos (limite superior) e mínimos (limite inferior) dos parâmetros codificados, de modo que a região de máximo rendimento fosse identificada no intervalo adotado. As variáveis 
em estudo codificadas e seus respectivos limites nominais encontram-se apresentados na Tabela 1.

TABELA 1- Limites codificados e valores nominais de trabalho das variáveis.

\begin{tabular}{c|c|c|c|c|c|c}
\hline \multirow{2}{*}{$\begin{array}{c}\text { Limites } \\
\text { Codificados }\end{array}$} & \multicolumn{3}{|c|}{ Rota Metílica } & \multicolumn{3}{c}{ Rota Etílica } \\
\cline { 2 - 7 } & $\begin{array}{c}\text { Hidróxido Sódio } \\
\text { (g) }\end{array}$ & $\begin{array}{c}\text { Metanol } \\
\text { PA } \\
(\mathbf{g})\end{array}$ & $\begin{array}{c}\text { Tempo } \\
\text { (min) }\end{array}$ & $\begin{array}{c}\text { Hidróxido } \\
\text { de Sódio } \\
(\mathbf{g})\end{array}$ & $\begin{array}{c}\text { Etanol } \\
\mathbf{9 8} \\
\mathbf{( g )} \mathbf{G L}\end{array}$ & $\begin{array}{c}\text { Tempo } \\
(\mathbf{m i n})\end{array}$ \\
\hline Inferior (-1) & 0,15 & 5,00 & 5,00 & 0,30 & 15,00 & 10,00 \\
\hline Central (0) & 0,25 & 10,00 & 15,00 & 0,50 & 20,00 & 20,00 \\
\hline Superior (+1) & 0,35 & 15,00 & 25,00 & 0,70 & 25,00 & 30,00 \\
\hline
\end{tabular}

Determinadas as variáveis de processo e seus limites de trabalho, obteve-se - Delineamento Composto Central Rotacionado com a definição das matrizes de planejamento experimental. As matrizes de planejamento para as rotas metílica e etílica são apresentadas na Tabela 2.

TABELA 2- Matriz de planejamento experimental das rotas de estudo.

\begin{tabular}{|c|c|c|c|c|c|c|}
\hline \multirow{2}{*}{ Experimentos } & \multicolumn{3}{|c|}{ Variáveis da Rota Metílica } & \multicolumn{3}{|c|}{ Variáveis da Rota Etílica } \\
\hline & $\begin{array}{c}\mathrm{NaOH} \\
\mathrm{X}_{1}\end{array}$ & $\begin{array}{c}\text { Metanol } \\
X_{2}\end{array}$ & $\begin{array}{c}\text { Tempo } \\
\mathbf{X}_{3}\end{array}$ & $\begin{array}{c}\mathrm{NaOH} \\
\mathrm{X}_{1}\end{array}$ & $\begin{array}{c}\text { Etanol } \\
X_{2}\end{array}$ & $\begin{array}{c}\text { Tempo } \\
\mathbf{X}_{3}\end{array}$ \\
\hline 1 & $0,15 \mathrm{~g}$ & $5,00 \mathrm{~g}$ & $5,00 \mathrm{~min}$ & $0,30 \mathrm{~g}$ & $15,00 \mathrm{~g}$ & $10,00 \mathrm{~min}$ \\
\hline 2 & $0,35 \mathrm{~g}$ & $5,00 \mathrm{~g}$ & $5,00 \mathrm{~min}$ & $0,70 \mathrm{~g}$ & $15,00 \mathrm{~g}$ & $10,00 \mathrm{~min}$ \\
\hline 3 & $0,15 \mathrm{~g}$ & $15,00 \mathrm{~g}$ & $5,00 \mathrm{~min}$ & $0,30 \mathrm{~g}$ & $25,00 \mathrm{~g}$ & $10,00 \mathrm{~min}$ \\
\hline 4 & $0,35 \mathrm{~g}$ & $15,00 \mathrm{~g}$ & $5,00 \mathrm{~min}$ & $0,70 \mathrm{~g}$ & $25,00 \mathrm{~g}$ & $10,00 \mathrm{~min}$ \\
\hline 5 & $0,15 \mathrm{~g}$ & $5,00 \mathrm{~g}$ & $25,00 \mathrm{~min}$ & $0,30 \mathrm{~g}$ & $15,00 \mathrm{~g}$ & $30,00 \mathrm{~min}$ \\
\hline 6 & $0,35 \mathrm{~g}$ & $5,00 \mathrm{~g}$ & $25,00 \mathrm{~min}$ & $0,70 \mathrm{~g}$ & $15,00 \mathrm{~g}$ & $30,00 \mathrm{~min}$ \\
\hline 7 & $0,15 \mathrm{~g}$ & $15,00 \mathrm{~g}$ & $25,00 \mathrm{~min}$ & $0,30 \mathrm{~g}$ & $25,00 \mathrm{~g}$ & $30,00 \mathrm{~min}$ \\
\hline 8 & $0,35 \mathrm{~g}$ & $15,00 \mathrm{~g}$ & $25,00 \mathrm{~min}$ & $0,70 \mathrm{~g}$ & $25,00 \mathrm{~g}$ & $30,00 \mathrm{~min}$ \\
\hline 9 & $0,25 \mathrm{~g}$ & $10,00 \mathrm{~g}$ & 1,80min & $0,50 \mathrm{~g}$ & $20,00 \mathrm{~g}$ & $36,20 \mathrm{~min}$ \\
\hline 10 & $0,25 \mathrm{~g}$ & $10,00 \mathrm{~g}$ & $31,80 \mathrm{~min}$ & $0,50 \mathrm{~g}$ & $20,00 \mathrm{~g}$ & $40,20 \mathrm{~min}$ \\
\hline 11 & $0,25 \mathrm{~g}$ & $1,60 \mathrm{~g}$ & $15,00 \mathrm{~min}$ & $0,50 \mathrm{~g}$ & $13,50 \mathrm{~g}$ & $20,00 \mathrm{~min}$ \\
\hline 12 & $0,25 \mathrm{~g}$ & $18,40 \mathrm{~g}$ & $15,00 \mathrm{~min}$ & $0,50 \mathrm{~g}$ & $26,40 \mathrm{~g}$ & $20,00 \mathrm{~min}$ \\
\hline 13 & $0,08 \mathrm{~g}$ & $10,00 \mathrm{~g}$ & $15,00 \mathrm{~min}$ & $0,24 \mathrm{~g}$ & $20,00 \mathrm{~g}$ & $20,00 \mathrm{~min}$ \\
\hline 14 & $0,42 \mathrm{~g}$ & $10,00 \mathrm{~g}$ & $15,00 \mathrm{~min}$ & $0,75 \mathrm{~g}$ & $20,00 \mathrm{~g}$ & $20,00 \mathrm{~min}$ \\
\hline 15 & $0,25 \mathrm{~g}$ & $10,00 \mathrm{~g}$ & $15,00 \mathrm{~min}$ & $0,50 \mathrm{~g}$ & $20,00 \mathrm{~g}$ & $20,00 \mathrm{~min}$ \\
\hline 16 & $0,25 \mathrm{~g}$ & $10,00 \mathrm{~g}$ & $15,00 \mathrm{~min}$ & $0,50 \mathrm{~g}$ & $20,00 \mathrm{~g}$ & $20,00 \mathrm{~min}$ \\
\hline 17 & $0,25 \mathrm{~g}$ & $10,00 \mathrm{~g}$ & $15,00 \mathrm{~min}$ & $0,50 \mathrm{~g}$ & $20,00 \mathrm{~g}$ & $20,00 \mathrm{~min}$ \\
\hline
\end{tabular}

${ }^{*} \mathrm{X}_{1}, \mathrm{X}_{2}$ e $\mathrm{X}_{3}$ são representações das variáveis de trabalho.

No preparo da matéria-prima realizou-se filtragem simples do óleo residual, visando à retirada de particulados suspensos que derivam do processo de fritura dos alimentos. Em seguida, preparou-se o catalisador, dissolvendo em béquer de 250 $\mathrm{mL}$ a massa do catalisador $(\mathrm{NaOH})$ na respectiva massa de álcool conforme as quantidades predeterminadas e apresentadas na Tabela 2.Na sequência, para cada experimento, uma alíquota de $50,00 \mathrm{~g}$ de óleo residual de fritura foi adicionada ao béquer de $250 \mathrm{~mL}$ contendo o catalisador previamente dissolvido no álcool, o béquer então foi levado ao banho ultrassônico, ultra-som a $40 \mathrm{kHz}$ e $70 \mathrm{~W}$ (Sanders Model, 
Soniclean 2 PS), permanecendo em reação por tempo equivalente ao experimento.

Após o término do experimento, a mistura foi transferida quantitativamente para um funil de separação de $200 \mathrm{~mL}$. Após o repouso de 24 horas para a completa separação de fases, a porção inferior (glicerina mais resto de catalisador e álcool) foi retirada do funil de separação e a fase sobrenadante (biodiesel mais impurezas), de coloração mais clara, foi lavada no próprio funil com $50 \mathrm{~mL}$ de água destilada a temperatura ambiente. Esse procedimento foi realizado quatro vezes para a máxima remoção do excesso de catalisador e demais impurezas hidrossolúveis do meio. $O$ material obtido foi então submetido às seguintes análises de caracterização físicoquímica: massa específica, ponto de entupimento de filtro a frio, índice de acidez e índice de iodo (insaturações).

A massa específica foi determinada pela técnica da picnometria. O ponto de entupimento de filtro a frio foi aferido via utilização de termopar (NBR 14747) sendo então registrada a temperatura de mínima fluidez. Os índices de acidez e iodo foram realizados de acordo com as normas técnicas especificadas pela ANP, NBR 14448 e EN ISO 1411, respectivamente. O tratamento dos resultados obtidos para essas caracterizações foi feito através do cálculo da média e do desvio padrão para cada análise realizada em triplicata.

\section{RESULTADOS E DISCUSSÃO}

Os rendimentos mássicos das rotas metílicas e etílicas da reação de transesterificação alcalina foram determinados após a lavagem e caracterização do produto obtido, estando os valores apresentados na Tabela 3.

TABELA 3 - Valores de rendimento mássico para o biodiesel obtido nas diferentes rotas experimentais.

\begin{tabular}{|c|c|c|c|c|c|}
\hline \multirow{2}{*}{ Experimentos } & \multicolumn{3}{|c|}{ Variáveis Codificadas } & \multicolumn{2}{|c|}{$\begin{array}{c}\text { Rendimentos Mássicos } \\
\mathrm{m} / \mathrm{m}(\%)\end{array}$} \\
\hline & $\begin{array}{c}\mathrm{NaOH} \\
\mathrm{X}_{1}\end{array}$ & $\begin{array}{c}\text { Álcool } \\
X_{2}\end{array}$ & $\begin{array}{c}\text { Tempo } \\
\mathrm{X}_{3}\end{array}$ & Rota Metílica & Rota Etílica \\
\hline 1 & $(-1)$ & $(-1)$ & $(-1)$ & 59,14 & 59,43 \\
\hline 2 & $(+1)$ & $(-1)$ & $(-1)$ & 72,47 & 64,29 \\
\hline 3 & $(-1)$ & $(+1)$ & $(-1)$ & 78,04 & 74,52 \\
\hline 4 & $(+1)$ & $(+1)$ & $(-1)$ & 88,78 & 88,46 \\
\hline 5 & $(-1)$ & $(-1)$ & $(+1)$ & 89,24 & 89,83 \\
\hline 6 & $(+1)$ & $(-1)$ & $(+1)$ & 85,92 & 84,28 \\
\hline 7 & $(-1)$ & $(+1)$ & $(+1)$ & 86,25 & 86,98 \\
\hline 8 & $(+1)$ & $(+1)$ & $(+1)$ & 85,38 & 85,87 \\
\hline 9 & $(0)$ & $(0)$ & $(-1,68)$ & 75,15 & 70,96 \\
\hline 10 & (0) & (0) & $(+1,68)$ & 92,35 & 88,98 \\
\hline 11 & (0) & $(-1,68)$ & (0) & 68,22 & 66,85 \\
\hline 12 & (0) & $(+1,68)$ & (0) & 85,45 & 82,79 \\
\hline 13 & $(-1,68)$ & (0) & (0) & 72,56 & 77,09 \\
\hline 14 & $(+1,68)$ & (0) & (0) & 85,63 & 77,42 \\
\hline 15 & (0) & (0) & (0) & 92,63 & 91,71 \\
\hline 16 & (0) & (0) & (0) & 92,47 & 91,64 \\
\hline 17 & (0) & (0) & (0) & 92,56 & 92,02 \\
\hline
\end{tabular}


Do rendimento obtido (variável resposta) em razão das quantidades de hidróxido de sódio, álcool (metanol ou etanol) e o tempo, variáveis independentes, obtiveram-se a regressão linear normal ao modelo matemático preditivo e as superfícies de respostas. As superfícies apresentam as regiões de máximo rendimento (em vermelho) contrapondo as variáveis abordadas no planejamento. A Figura 1 representa as superfícies obtidas para a reação de transesterificação alcalina em rota metílica.

a)

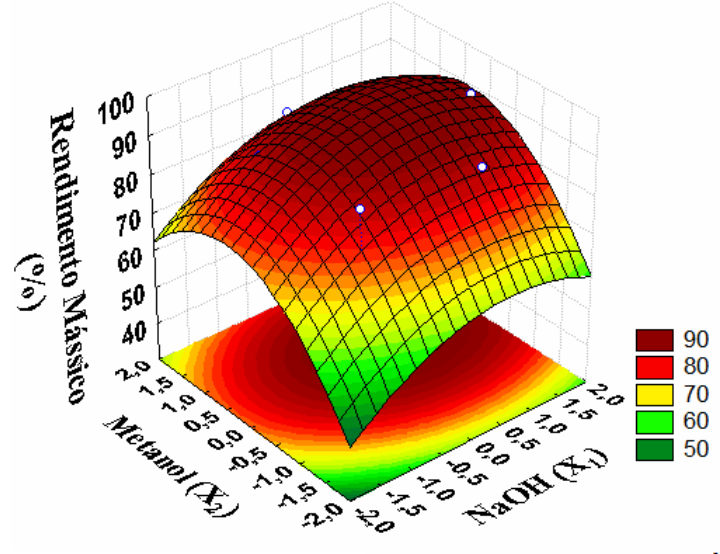

b)

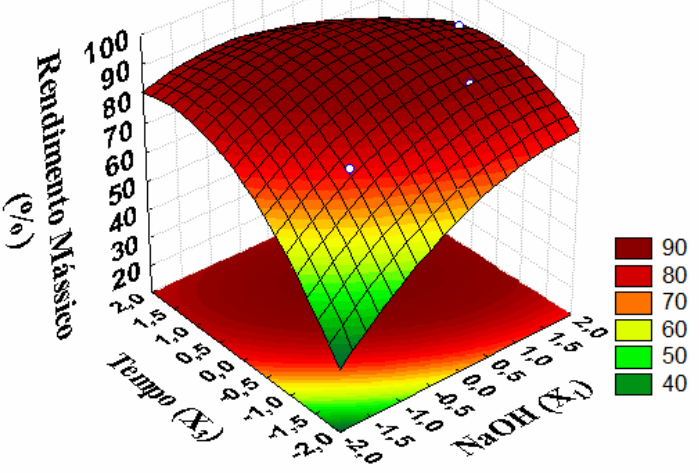

c)

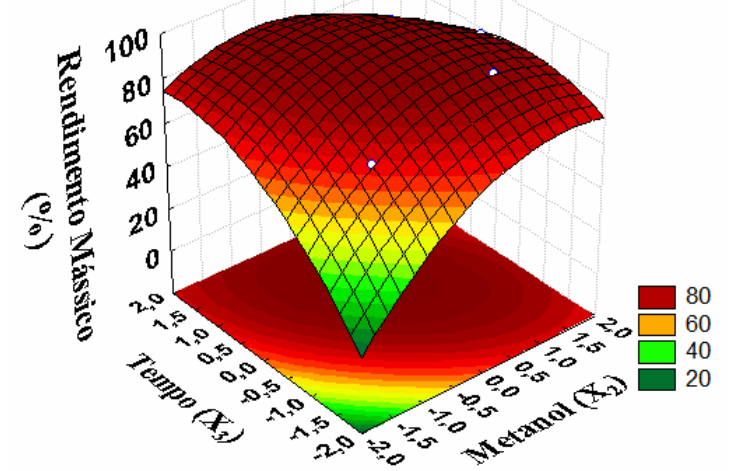

|FIGURA 1- Superfícies de respostas da reação de transesterificação metílica do máximo rendimento em função das quantidades: (a) $\mathrm{NaOH}$ e Metanol, (b) $\mathrm{NaOH}$ e Tempo, (c) Metanol e Tempo.

Fonte: Autores, 2017.

Na Figura 1a, observa-se o comportamento parabólico para os eixos do metanol e $\mathrm{NaOH}$, indicando a interação entre as duas variáveis. Em baixas quantidades de metanol e $\mathrm{NaOH}$ a formação do íon alcóxido $\left(\mathrm{CH}_{3} \mathrm{O}^{-}\right)$não foi suficiente para promover altos rendimentos. No outro extremo, as maiores quantidades de metanol e $\mathrm{NaOH}$ favoreceram a reação de saponificação, diminuindo assim o rendimento mássico do biodiesel. Resultados semelhantes foram obtidos por Canesin et al. (2014) na reação de transesterificação alcalina de óleo residual de soja.

Nas Figuras 1b e 1c, o comportamento das variáveis apresentou-se em forma de curva linear, os maiores rendimentos da reação de transesterificação ocorreram para as quantidades médias de tempo e $\mathrm{NaOH}$, tempo e metanol, respectivamente. 
Houve um decréscimo no rendimento para os valores próximos do limite superior, uma vez que, o maior tempo reacional e a umidade oriunda dos óleos residuais de fritura e favorecem a hidrólise dos mono-álquil ésteres (biodiesel) formados na reação de transesterificação ( LI et al., 2012).

O ajuste dos coeficientes proposto pelo software Statistica®, retornou o modelo matemático com índice de ajuste de $98 \%$ aos dados experimentais (valores preditos pelos observados), valor semelhante ao encontrado por Melo et al. (2011). Os valores otimizados para a síntese de biodiesel em rota metílica foram; 0,20 g de $\mathrm{NaOH}, 9,32 \mathrm{~g}$ de metanol e 13 minutos. Os rendimentos médios no ponto de ótimo foram de aproximadamente $92 \%$.

De forma análoga ao realizado para a rota metílica, as superfícies de respostas foram obtidas para a rota etílica da reação de transesterificação alcalina do óleo residual de fritura (Figura 2).

a)

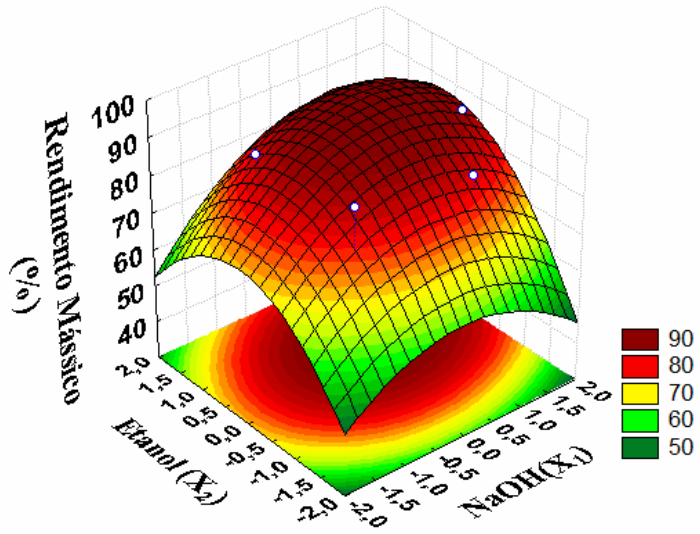

b)

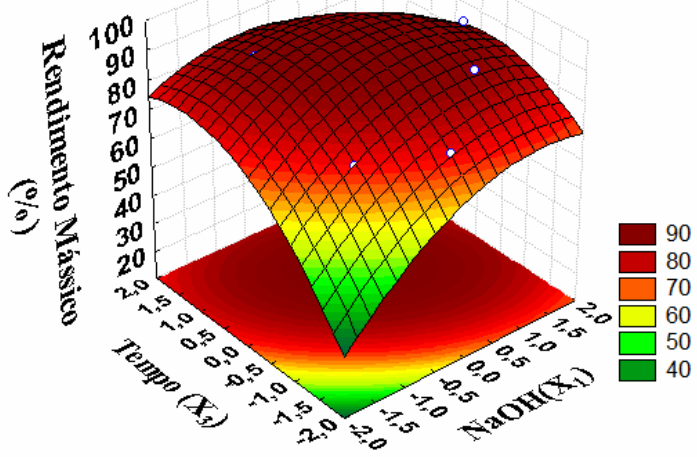

c)

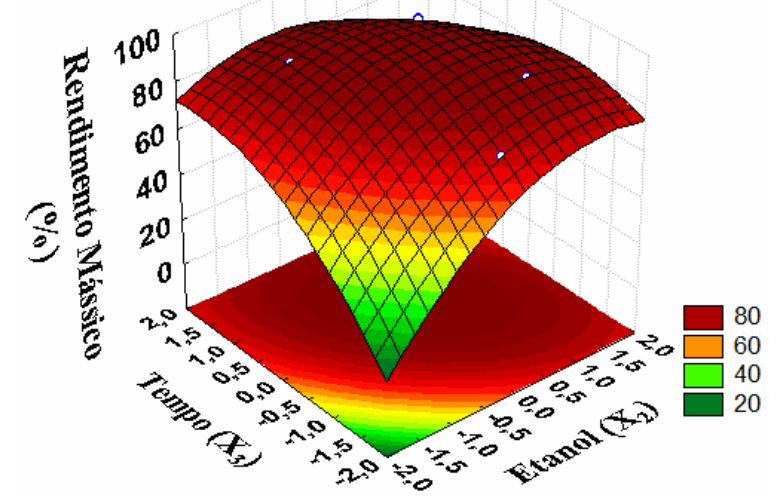

FIGURA 2- Superfícies de respostas da transesterificação etílica do máximo rendimento em função: (a) NaOH e Etanol, (b) NaOH e Tempo, (c) Etanol e Tempo.

Fonte: Autores, 2017.

A interação entre o etanol e o $\mathrm{NaOH}$ (Figura 2 a) foi semelhante a observada na rota metílica $\mathrm{NaOH}$ (Figura 1a). O decréscimo no rendimento mássico de biodiesel para as maiores quantidades de $\mathrm{NaOH}$ ocorreu devido reação de saponificação. Resultados semelhantes foram relatados por Cunha Júnior et al. (2014) na reação de transesterificação alcalina em rota etílica empregando óleo residual de fritura.

$\mathrm{Na}$ Figura 2b, o maior rendimento foi observado na região central do intervalo ENCICLOPÉDIA BIOSFERA, Centro Científico Conhecer - Goiânia, v.14 n.26; p. 1459 
variável, com valores crescentes proporcionais ao aumento do $\mathrm{NaOH}$ e do tempo reacional. O decréscimo do rendimento foi observado nos limites superiores dessas variáveis, já que a maior basicidade do meio favoreceu a reação de saponificação (LÔBO et al., 2009).

Em relação às variáveis etanol e tempo reacional (Figura $2 \mathrm{c}$ ), observou-se que o rendimento mássico de biodiesel foi reduzido em função do incremento dessas variáveis. O excesso de etanol no final do processo dificulta a decantação da glicerina no funil de separação, dificultando assim a lavagem do biodiesel e posterior separação conforme observaram Silva Júnior et al. (2009) na transesterificação etanólica do óleo de babaçu.

A regressão do modelo apresentou ajuste de $97 \%$ dos valores preditos aos observados, com valores médios de rendimento máximo em 90,35\%. A região otimizada ocorreu com $0,39 \mathrm{~g}$ de $\mathrm{NaOH} ; 17,35 \mathrm{~g}$ de etanol e tempo de reação igual a 20 minutos. A diferença encontrada nos rendimentos mássicos das rotas metílica $(92 \%)$ e etílica $(90,35 \%)$ se deve ao íon metóxi oriundo do metanol, ser um nucleófilo mais forte que o íon etóxi oriundo do etanol. A força do nucléofilo também interferiu na cinética da reação de transesterificação, por isso, o tempo reacional otimizado da rota etílica, em banho ultrassônico, foi superior (35\%) ao tempo reacional da rota metílica.

O gráfico de Pareto apresentado na Figura 3 evidencia os parâmetros de maior influência no rendimento do biodiesel para as rotas metílica (Figura 3a) e etílica (Figura 3b).

a)

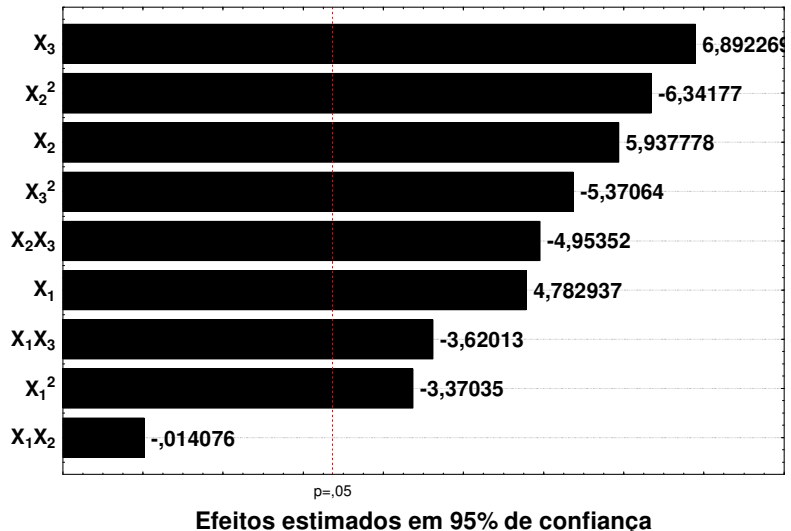

FIGURA 3-Gráfico de Pareto para as transesterificação etílica. b)

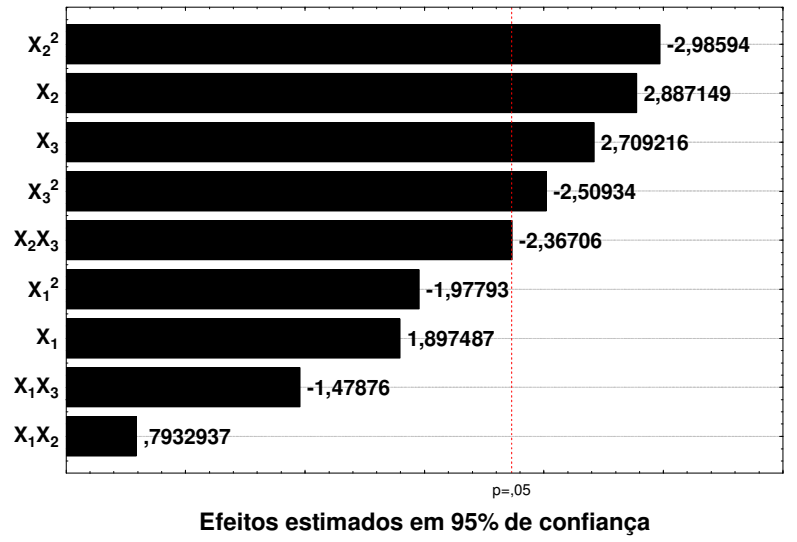

(a) transesterificação metílica, (b)

Fonte: Autores, 2017.

$\mathrm{Na}$ rota metílica (Figura 3a), o tempo foi a variável de maior efeito positivo, seguido da influência do metanol e $\mathrm{NaOH}$, conforme observado também no comportamento das superfícies de resposta (Figura 1). Ruschel et al. (2016) observaram, em estudos sobre a produção de biodiesel a partir de óleo de amendoim, que o tempo e a quantidade de álcool são as variáveis de maior influência na otimização do processo. A relação quadrática das variáveis apresentou efeito negativo para o intervalo adotado, com nível de confiança de 95\%.

$\mathrm{Na}$ rota etílica (Figura $3 \mathrm{~b}$ ) a influência negativa quadrática do etanol $98^{\circ} \mathrm{GL}$ foi a mais significativa, esse resultado corrobora com a hipótese de que o excesso de etanol no final da reação de síntese do biodiesel dificulta a separação da glicerina, 
reduzindo assim o rendimento do processo conforme foi observado na superfície de resposta (Figura 2c). O tempo de reação apresentou também interação quadrática negativa. Shuit et al. (2013) relataram que a variável tempo influi também de forma negativa no rendimento do biodiesel. $\mathrm{O}$ maior tempo reacional associado à umidade do meio favoreceu a hidrólise dos mono-álquil ésteres (biodiesel) formados no processo de transesterificação alcalina do óleo residual de fritura em rota etílica.

Com os valores das reações otimizadas, a caracterização físico-química das triplicatas no ponto central foram caracterizadas e são apresentadas na Tabela 4. Também foram apresentados, para fim de comparação, os referidos valores estabelecidos pela RESOLUÇÃO № 45 da ANP de 25 de agosto de 2014 para o biodiesel sem mistura com diesel (B100).

TABELA 4 - Resultados das análises físico-químicas para as amostras de biodiesel produzidas no valor de máximo rendimento.

\begin{tabular}{|c|c|c|c|c|c|}
\hline & Amostras & $\begin{array}{c}\text { Densidade } \\
\text { à } 20^{\circ} \mathrm{C} \text { do } \\
\text { biodiesel } \\
\left(\mathrm{g} . \mathrm{cm}^{-3}\right)\end{array}$ & $\begin{array}{l}\text { Ponto de } \\
\text { entupimento } \\
\text { de filtro a } \\
\text { frio }\left({ }^{\circ} \mathrm{C}\right)\end{array}$ & $\begin{array}{c}\text { Índice de } \\
\text { acidez } \\
\text { (mg de } \\
\text { KOH.g } \text { (m) }^{-1}\end{array}$ & $\begin{array}{l}\text { Índice de } \\
\text { lodo }(\mathrm{g} \text { de } \\
\left.\mathrm{I}_{2} / 100 \mathrm{~g}\right)\end{array}$ \\
\hline \multirow{3}{*}{$\begin{array}{c}\text { Rota } \\
\text { Metílica }\end{array}$} & 01 & $0,87 \pm 0,01$ & $4,0 \pm 0,4$ & $0,12 \pm 0,02$ & $0,650 \pm 0,020$ \\
\hline & 02 & $0,87 \pm 0,01$ & $4,0 \pm 0,5$ & $0,12 \pm 0,01$ & $0,680 \pm 0,021$ \\
\hline & 03 & $0,86 \pm 0,01$ & $5,0 \pm 0,5$ & $0,11 \pm 0,02$ & $0,660 \pm 0,021$ \\
\hline \multirow{3}{*}{$\begin{array}{c}\text { Rota } \\
\text { Etílica }\end{array}$} & 01 & $0,87 \pm 0,01$ & $6,0 \pm 0,5$ & $0,15 \pm 0,02$ & $0,774 \pm 0,018$ \\
\hline & 02 & $0,87 \pm 0,01$ & $5,0 \pm 0,5$ & $0,13 \pm 0,01$ & $0,790 \pm 0,022$ \\
\hline & 03 & $0,88 \pm 0,01$ & $5,0 \pm 0,5$ & $0,12 \pm 0,01$ & $0,802 \pm 0,020$ \\
\hline $\begin{array}{c}\text { Valores } \\
\text { estabelecidos } \\
\text { pela ANP }\end{array}$ & & 0,85 e 0,90 & $\begin{array}{l}\text { Até } 10 \text { (varia } \\
\text { de acordo } \\
\text { com a região) }\end{array}$ & Até 0,50 & $<115$ \\
\hline
\end{tabular}

Após análise conjunta das caracterizações físico-químicas (Tabela 4) verificouse que os resultados obtidos foram condizentes com os limites estabelecidos pela ANP. Com isso, o produto obtido no ponto de ótimo pode ser classificado como biodiesel de acordo com os parâmetros de densidade, ponto de entupimento de filtro a frio e índices de acidez e iodo.

Valores semelhantes do ponto de entupimento de filtro a frio e índice de acidez também foram observados por Sulaiman et al. (2014). Vale destacar que Canesin et. al. (2014) observaram em estudos de síntese de biodiesel a partir de óleo de soja refinado valores de índice de iodo maiores (5\%) que os observados no presente estudo. Essa variação ocorre porque os óleos virgens apresentam em suas estruturas maior número de insaturações nos triglicerídeos em relação aos óleos residuais de fritura, uma vez que estes sofrem reações de adição (reduzem as insaturações) com o aumento de temperatura (LÔBO et al., 2009; PRASERTSIT et al., 2013).

Para o cálculo dos custos de produção para um litro do biodiesel, consideraram-se os valores médios dos produtos como sendo os praticados no ano de 2017 (janeiro-setembro) bem como a recuperação do catalisador alcóxido em $50 \%$. Sendo assim, o litro de biodiesel pela rota metílica foi valorado em $R \$ 2,124$ enquanto que o litro para a rota etílica foi de $\mathrm{R} \$ 2,453$. O maior custo de produção para a rota etílica $(13,41 \%)$ é justificado principalmente pelo maior tempo de reação $(34,40 \%)$ em relação à rota metílica. Os custos de produção nas rotas etílicas e 
metílicas ficaram inferiores aos valores comerciais praticados no mercado para o litro de diesel comum $R \$ 3,149$ (ANP, setembro de 2017).

Os biodieseis obtidos pelas rotas otimizadas além da vantagem ambiental, também se mostraram economicamente vantajosos do ponto de vista de produção. Barros et al. (2006) estimaram que para plantas de até 100 mil toneladas ano de biodiesel, os custos de processo podem ser reduzidos em até $40 \%$ considerando 0 reciclo energético, o reaproveitamento dos subprodutos e a escala industrial.

\section{CONCLUSÃO}

O resultado da produção maximizada de biodiesel a partir de óleo residual de fritura, com o auxílio da técnica de superfícies de resposta, se mostrou satisfatória, a caracterização do produto obtido apresentou consonância com os principais parâmetros estabelecidos pela ANP.

Os rendimentos obtidos para a reação otimizada de transesterificação alcalina nas rotas etílica e metílica foram superiores a 90\%. Esses valores de rendimento são relevantes, quando se trata de uma matéria prima tão complexa e diversificada como o óleo residual de fritura.

O custo por litro do biodiesel obtido no banho ultrassônico nas rotas etílica e metílica foi inferior ao valor de mercado para o diesel de petróleo. Nesse sentido, destaca-se o potencial dessa metodologia para a redução dos custos com a síntese de energia renovável, o destino final ambientalmente adequado do óleo residual de fritura e a maximização da produção de biodiesel em escala industrial.

\section{REFERÊNCIAS}

AARTHY, M.; SARAVANAN, P.; GOWTHAMAN, M. K.; ROSE, N.; KAMINI, N. R. Enzymatic transesterification for production of biodiesel using yest lipases: $\mathrm{Na}$ overviee. Chemical Engineering Research and Design, v. 92, n.8, p.1591-1601, 2014. Disponível em <https://doi.org/10.1016/j.cherd.2014.04.008>doi: 10.1016/j.cherd.2014.04.008.

ANP (2014) Agência Nacional de Petróleo, gás natural e Biocombustíveis. Preços dos combustíveis. Disponível em: http://www.anp.gov.br/index.asp. Acesso em 03 de setembro de 2017.

ANP - Agência Nacional de Petróleo, gás natural e Biocombustíveis. Portaria ANP $n^{\circ}$ 255, de 15 de setembro de 2003. Estabelece a especificação do biodiesel puro a ser adicionado ao óleo diesel automotivo para testes em frotas cativas ou para uso em processo industrial específico nos termos da Portaria ANP no 240, de 25 de agosto de 2003. Brasília: ANP, 2003. Disponível em: < https://www.legisweb.com.br/legislacao/?id=185852>. Acesso em: 03 set. 2017.

BARROS, G.S.C.; SILVA, A.P.; PONCHIO, L.A.; ALVES, L.R.; OSAKI, M. CENAMO,M. Custos de produção de biodiesel no Brasil. Revista de Política Agrícola.v.15. n.3.p-36-50, 2006.2 Disponível em: https://seer.sede.embrapa.br/index.php/RPA/article/view/508.

BRUNSCHWIG, C.; MOUSSAVOU, W.; BLIN, J. Use of bioethanol for biodiesel production.Progress in Energy and Combustion Science, v.38, n.2, p.283-301, 2012.Disponívelem<https://doi.org/10.1016/j.pecs.2011.11.001>.doi: 
10.1016/j.pecs.2011.11.001.

CANESIN, E.A.; DE OLIVEIRA, C.C.; MATSUSHITA, M.; DIAS, L.F.; PEDRÃO, M.R.; SOUZA, N.E. Characterization of residual oils for biodiesel production. Electronic Journal of Biotechnology, v.17, p.39-45, 2014. Disponível em: http://dx.doi.org/10.1016/j.ejbt.2013.12.007. doi: 10.1016/j.ejbt.2013.12.007.

COSTA, J.F.; ALMEIDA, M.F.; ALVIM-FERRAZ, M.C.M.; DIAS, J.M. Biodiesel production using oil from fish canning industry wastes. Energy Conversion and Management. v.74, p.17-23, 2013. Disponível em: http://dx.doi.org/10.1016/j.enconman.2013.04.032.doi:

10.1016/j.enconman.2013.04.032

CREMONEZ, A. P.; FEROLDI, M.; NADALETI, W.C; ROSSI, E.; FEIDEN, A.; CAMARGO, M.P.; CREMONEZ, F.E.; KLAJN, F.F. Biodiesel production in Brazil: Current scenario and perspectives. Renewable sustainable energy Rev, v.42, p.415-428, 2014.Disponível em: http://www.sciencedirect.com/science/article/pii/S1364032114008211?via\%3Dihub. doi.org/10.1016/j.rser.2014.10.004. doi: 10.1016/j.rser.2014.10.004

CUNHA JÚNIOR, A.; FEDDERN, V.; DE PRÁ, M.C.; HIGARASHI, M.M.; ABREU, P.G.; COLDEBELLA, A. Synthesis and characterization of ethylic biodiesel from animal fat wastes. Fuel , v.105, p.228-234, 2013. Disponível em: http://dx.doi.org/10.1016/j.fuel.2012.06.020. doi: 10.1016/j.fuel.2012.06.020

EN 14111, Fat and oil derivates-Fatty acid methyl esters (FAME)- Determination of lodine value, European Committee for Standardization, 2003. Disponível em: https://shop.bsigroup.com/ProductDetail/?pid=000000000030084518. Acesso em 03 de setembro de 2017.

GOLE, V. L.; NAVEEN, K.R.; GOGATE, P.R. Hydrodynamic cavitation as an efficient approach for intensification of synthesis of methyl esters from sustainable feedstock. Chem Eng Process Process Intensif . v. 71, p.70--76, 2013. Disponível em: https://doi.org/10.1016/j.cep.2012.10.006. doi: 10.1016/j.cep.2012.10.006.

JIANBING, J.; WANG, J.; YONGCHAO, L.; YUNLIANG, Y.; ZICHAO, X. Preparation of biodiesel with the help of ultrasonic and hydrodynamic cavitation. Ultrasonics, v.44, p.411-414, 2006. Disponível em <https://doi.org/10.1016/j.ultras.2006.05.020>.doi: 10.1016/j.ultras.2006.05.020.

KELKAR, M. A.; GOGAT, P. R.; PANDIT, A. B. Intensification of esterification of acids for synthesis of biodiesel using acoustic and hydrodynamic cavitation. Ultrasonics Sonochemistry, v.15, n. 3, p.188-194, 2008. Disponível em <https://doi.org/10.1016/j.ultsonch.2007.04.003>.doi: 0.1016/j.ultsonch.2007.04.003.

KIAKALAIEH, A.T.; AMIN, N.A.S.; MAZAHERI, H. A review on novel processes of biodiesel production from waste cooking oil. Applied Energy.n.104, p.683-710, 2013. Disponível em: http://dx.doi.org/10.1016/j.apenergy.2012.11.061.doi: 10.1016/j.apenergy.2012.11.061 
LI, J.; FU, Y.; J.; QU, X.; J., WANG, W.; LUO, M.; ZHAO, C. J.; ZU, Y. G. Biodiesel productionfromyellowhorn (Xanthocerassorbifolia Bunge.) seed oil using ionex changeres in as heterogeneous catalyst. Bioresource technology, v. 108, p. 112118, 2012. Disponível em <https://doi.org/10.1016/j.biortech.2011.12.129>.doi: 10.1016/j.biortech.2011.12.129.

LÔBO, I. P.; FERREIRA, S. L. C.; CRUZ, S. R. Biodiesel: parâmetros de qualidade e métodos analíticos. Química Nova, v. 32, n.6, p.1596-1608, 2009. Disponível em <http://dx.doi.org/10.1590/S0100-40422009000600044>.doi:

10.1590/S010040422009000600044.

LOPEZ, D.E.; GOODWIN, J.G.; BRUCE, D.A. Transesterification of triacetin with ethanol on Nafion acid resins. J Catal . 245 (2): p.381-391, 2007.Disponível em: https://www.researchgate.net/publication/222693085_Transesterification_of_triacetin with_methanol_on_solid_acid_and_base_catalysts doi: 10.1016/j.supflu.2010.12.007

MADDIKERI, G.L.; GOGATE, P.R.; PANDIT, A.B. Intensified synthesis of biodiesel using hydrodynamic cavitation reactors based on the interesterification of waste cooking oil. Fuel. v.137, p. 285-292, 2014. Disponível em: <http://dx.doi.org/10.1016/j.fuel.2014.08.013>. doi: 0016-2361/ 2014

MASON, T. Sonochemistry andt heenvironment - Providing a "green" link between chemistry, physicsan dengineering. Ultrasonics Sonochemistry, v. 14, n.4, p. 476483, 2007. Disponível em <https://doi.org/10.1016/j.ultsonch.2006.10.008>.doi: 10.1016/j.ultsonch.2006.10.008.

MELO, T. C.; BEGNINI, M. L.; FERREIRA, D. C. Estudo paramétrico das variáveis envolvidas na reação de transesterificação etílica do sebo bovino. Enciclopédia Biosfera, v.7, n.13, p. 1821-1832, 2011.Disponível em: http://www.conhecer.org.br/enciclop/2011b/engenharias/estudo\%20parametrico.pdf.

MOTA, J. A.; SILVA, X. A.; GONCALVES, L. C. Gliceroquímica: novos produtos e processos a partir da glicerina de produção de biodiesel. Química Nova, São Paulo , v. 32, n. 3, p. 639-648, 2009. Disponível em<http://dx.doi.org/10.1590/S010040422009000300008 >.doi: 10.1590/S0100-40422009000300008.

\section{NBR 14448, Produtos de Petróleo-Determinação do índice de acidez pelo método de titulação potenciométrico, 2001. Disponível em: https://www.target.com.br/previewer-v3/Viewer.asp?nbr=38326\&token=2dbd1c28- c479-4d4e-a18d-6353e756e41d\&sid=5qprl5ovlsnoaxxpz20tya1n\&. Acesso em 03 de setembro de 2017.}

NBR 14747 -Óleo diesel-Determinação da Temperatura do ponto de entupimento de filtro a frio, 2015. Disponível em: https://www.target.com.br/previewer-v3/Viewer.asp?nbr=38359\&token=2dbd1c28c479-4d4e-a18d-6353e756e41d\&sid=5qprl5ovlsnoaxxpz20tya1n\&. Acesso em 03 de setembro de 2017. 
PAULA, C. D.; BARROS, F. S.; CORREIA, L. M.; VIEIRA, R. S. Avaliação de catalisador a base de conchas de ostras para a produção de biodiesel utilizando planejamento fatorial. Holos, v. 1, p. 316-324, 2017. Disponível em <http://dx.doi.org/10.15628/holos.2017.5204>.doi: 10.15628/holos.2017.5204.

PRASERTSIT, K.; MUEANMAS, C.; TONGURAI, C. Transesterification of palm oil with methanol in a reactive distillation column. Chem Eng Process Process Intensif. $\quad$ v.70, p.21-26, $2013 . \quad$ Disponível em: https://doi.org/10.1016/j.cep.2013.05.011. doi: 10.1016/j.cep.2013.05.011

REIS, M. C.; FREITAS, F.A.; LACHTER, E.R.; GIL, R.A.S.S.; NASCIMENTO, R.S.V.; POUBEL, R.L.; BORRÉ, L.B. Produção de biodiesel a partir de ácidos graxos provenientes do refino de óleos vegetais via catálise ácida heterogênea e microondas. Química Nova, v.38, n.10, p.1307-1312, 2015. Disponível em: $<$ http://www.scielo.br/scielo.php?script=sci_arttext\&pid=S0100-

$40422015001001307 \& \operatorname{lng}=e n \& n r m=i s o>$. . doi: 10.5935/0100-4042.20150163.

RUSCHEL, C. F.; FERRÃO, M. F.; SANTOS, F. P.; SAMIOS, D. Otimização do processo de transesterificação em duas etapas para produção de biodiesel através do planejamento experimental de Doehlert. Química Nova, v.39, n. 3, p.267-272, 2016. Disponível em <http://dx.doi.org/10.5935/0100-4042.20160018>. doi: 10.5935/0100-4042.20160018.

SADEGHINEZHAD, E.; KAZI, S.N.; SADEGHINEJAD F.; BADARUDIN, A.; MEHRALI, M.; SADRI, R.; SAFAEI, M.R. A comprehensive literature review of biofuel performance in internal combustion engine ad relevant costs involvement. Renew Sust Energ Rev.30:29-44, 2014. Disponível em: http://www.academia.edu/7946023/A_Comprehensive_Literature_review_of_Biofuel_ Performance_in_Internal_combustion_Engine_and_relevant_Costs_involvement doi: 10.1007/s10098-014-0849-X

SHUIT, S. H.; YEE, K. F.; LEE, K. T.; SUBHASH, B.; TAN, S. H.; Evolution towards the utilization off unction alised carbon nanotubes as a new generation catalyst support in biodiesel production: an over view. RSC Advances. n.3, p. 9070-9094, 2013. Disponível em <http://dx.doi.org/10.1039/C3RA22945A>.doi: 10.1039/C3RA22945A

SILVA JUNIOR, O.; SILVA, R. B.; DA SILVA, C. C.; SOARES, L. S. Biodiesel de babaçu (Orbignya sp.) obtido por via etanólica. Quimica Nova, v.30, n.3, p.600-603, 2009. Disponível em: http://dx.doi.org/10.1590/S0100-40422007000300019 doi: 10.1590/S0100-40422007000300019.

SNEHA, E.; MAHES, A. R.; MEERA, S.; BEGUM, A. N. Energy Conversion and Management. n.91 442-450, $2015 . \quad$ Disponível https://doi.org/10.1016/j.jtuxci.2015.05.006. doi: 10.1016/j.jtuxci.2015.05.006.

SULAIMAN, S.; KHAIRUDIN, N.; JAMAL, P.; ALAM, Z. Z.; AZMI, S. Characterization off is hbonecatalyst for biodiesel production. International Journal of Biological, Biomolecular, Agricultural, Food and Biotechnological Engineering, v.8, n.5, 476-478, 2014. Disponível em <http://dx.doi.org/10.9767/bcrec.11.2.556.245249>.doi: 10.9767/bcrec.11.2.556.245-249. 